\title{
New diagnostic techniques and technologies at JET (invited)
}

A. Murari, B. Alper, L. Bertalot, J. H. Brzozowski, P. Coad, S. Conroy, E. De La Luna, G. Ericsson, H. G. Esser, S. Hacquin, J. Kaellne, V. Kiptily, K. Lawson, M. O'Mullane, V. Philipps, S. Sharapov, K.-D. Zastrow, and JETEFDA Contributors

Citation: Review of Scientific Instruments 77, 10F529 (2006);

View online: https://doi.org/10.1063/1.2336447

View Table of Contents: http://aip.scitation.org/toc/rsi/77/10

Published by the American Institute of Physics

\section{Articles you may be interested in}

Enhanced core charge exchange recombination spectroscopy system on Joint European Torus

Review of Scientific Instruments 77, 10F102 (2006); 10.1063/1.2222170

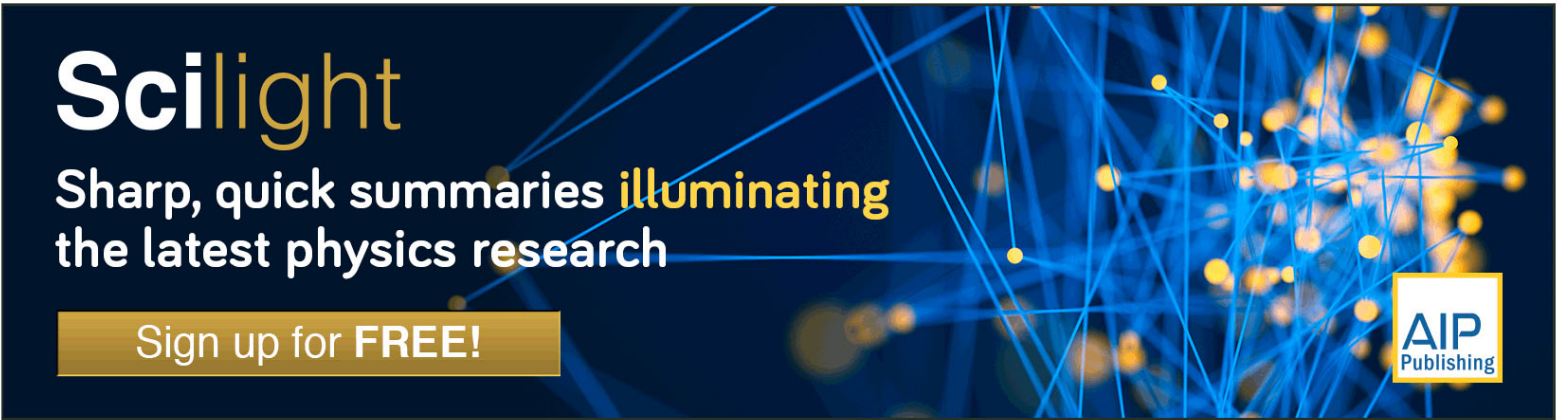




\section{New diagnostic techniques and technologies at JET (invited)}

A. Murari

Associazione EURATOM-ENEA per la Fusione, Consorzio RFX, 4-35127 Padova, Italy

B. Alper

EURATOM/UKAEA Fusion Association, Culham Science Centre, Abingdon OX14 3DB, United Kingdom

L. Bertalot

Associazione EURATOM-ENEA per la Fusione, CR ENEA Frascati, 00044 Frascati, Roma, Italy

J. H. Brzozowski

Alfvén Laboratory, KTH, EURATOM-VR Association, SE-10044 Stockholm, Sweden

P. Coad

EURATOM/UKAEA Fusion Association, Culham Science Centre, Abingdon OX14 3DB, United Kingdom

S. Conroy

INF, Uppsala University, EURATOM-VR Association, Box 535, 75121 Uppsala, Sweden

E. De La Luna

Asociación EURATOM-CIEMAT para Fusión, CIEMAT, Avenida Complutense, 22 E-28040 Madrid, Spain

G. Ericsson

INF, Uppsala University, EURATOM-VR Association, Box 535, 75121 Uppsala, Sweden

H. G. Esser

Institut für Plasmaphysik, Forschungszentrum Jülich, Association EURATOM-FZJ, Trilateral Euregio Cluster, D-52425 Jülich, Germany

S. Hacquin

CFN, Associacao IST/EURATOM, 1049-001 Lisboa, Portugal

J. Kaellne

INF, Uppsala University, EURATOM-VR Association, Box 535, 75121 Uppsala, Sweden

V. Kiptily and K. Lawson

EURATOM/UKAEA Fusion Association, Culham Science Centre, Abingdon OX14 3DB, United Kingdom

M. O'Mullane

Department of Physics and Applied Physics, University of Strathclyde, Glasgow G4 ONG, United Kingdom

V. Philipps

CFN, Associacao IST/EURATOM, 1049-001 Lisboa, Portugal

S. Sharapov and K.-D. Zastrow

EURATOM/UKAEA Fusion Association, Culham Science Centre, Abingdon OX14 3DB, United Kingdom

JET-EFDA Contributors ${ }^{\text {a) }}$

(Received 9 May 2006; presented on 11 May 2006; accepted 16 July 2006;

published online 23 October 2006)

The European Fusion Development Agreement's mission for JET is the development of ITER scenarios exploiting the specific properties of the device. This task requires significant improvements in the measuring techniques. The most innovative diagnostic upgrades are in the fields of edge measurements, detection of fast magnetohydrodynamics modes and "burning plasma" diagnostics. The importance of plasma-wall interactions, and, in particular, the issue of tritium inventory promoted the development of the quartz microbalance, a detector with improved time resolution to measure material redeposition in the remote areas of the inner divertor. Measurement of Alfvén cascades with unprecedented spectral resolution, reaching a toroidal $n$ number of up to 16 , was obtained using an $O$-mode microwave reflectometer as an interferometer. For the diagnosis of the fusion products, a new approach is being developed to measure the He ash based on double charge exchange between thermalized particles and neutrals from JET beams. There have been several upgrades of the neutron diagnostic systems, one of which, the new time of flight neutron spectrometer designed for high counting rates is described. () 2006 American Institute of Physics. [DOI: 10.1063/1.2336447]

\footnotetext{
${ }^{a)}$ See Annex of J. Pamela et al., Overview of Recent JET Results, Nucl. Fus. 43 (2003).
} 


\section{INTRODUCTION}

The mission of JET under EFDA is centered on advancing the development of operating scenarios and testing technologies for ITER. Plasma scenarios, such as "ELMy $H$-mode" and "advanced scenarios" are the leading contenders under development. The former is characterized by the formation of a transport barrier at the plasma edge, which results in improved confinement, as documented in many devices. Unfortunately this increase in confinement is accompanied by edge instabilities, known asedge localized modes (ELMs), which, if not controlled better than in present day machines, would induce excessive power loads on ITER divertor. With an advanced scenario-type discharge, the transport barriers occur deeper in the plasma, thus raising the possibility for steady state operation since the plasma current is generated by the pressure gradients (bootstrap effect) rather than the pulsed transformer action used now. The ITER scenario development program at JET consists of optimizing these configurations and exploring the limits of the operational space in the most ITER-relevant configuration achievable. A substantial program of diagnostic developments, covering the full range of plasma parameters from the edge to the core, is necessary to support the general scientific mission of the experiment.

A significant part of the research effort at JET has been devoted to the plasma wall interactions, in order to improve plasma performance. Such optimization is, of necessity, constrained by the wall materials available, principally carbon in JET. Particular attention has been devoted in the last few years to the study of erosion, redeposition, and tritium retention, using new diagnostics with better time resolution which are capable of providing the necessary information on, at least, a shot to shot basis and not relying simply on post mortem analysis (see following section). The capabilities of JET to operate with tritium and to accelerate ${ }^{4} \mathrm{He}$ using powerful electromagnetic waves in the radiofrequency range give the opportunity to investigate various "burning plasma" issues. One such issue is the effect of magnetohydrodynamics (MHD) instabilities on fast particles and their confinement. A series of new systems and analysis techniques was developed to improve the detection of MHD instabilities, with particular attention to the Alfvén modes, given their potential to affect alpha particle confinement and to provide useful diagnostic information, particularly about the current profile (see Sec. III). Other aspects of burning plasma physics including $\mathrm{He}$ ash detection, fast particle measurements, and neutron spectrometry have also been addressed in the past few years. In the current program, particular attention is being devoted to slowing down and thermalized alpha particles, whose detection is particularly challenging from a diagnostic point of view (see Sec. IV). A new neutron spectrometer, called time of flight for optimized rate (TOFOR), was installed during the last shutdown, to provide routinely the spectra of the 2.45 MeV neutrons (see Sec. V). Conclusions and comments on future prospects are the subject of Sec. VI.

\section{DIAGNOSTICS FOR EROSION AND REDEPOSITION}

Ameliorating the power load on plasma facing components will be one of the major technological problems for

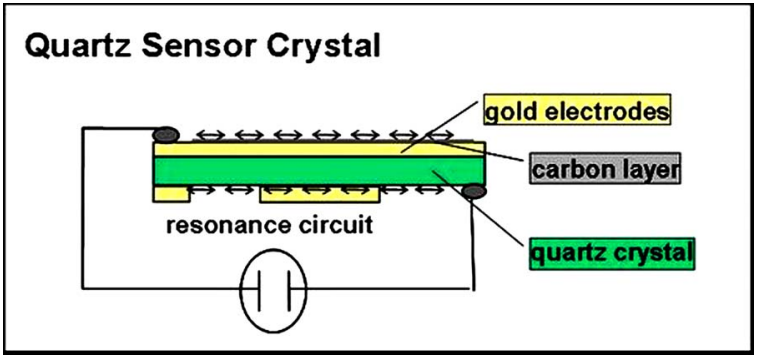

FIG. 1. Quartz microbalance: principle of operation. The resonant circuit oscillates at different frequencies depending on the amount of material deposited on the crystal.

ITER as well as for later reactors. The need to guarantee adequate lifetime of the presently available materials imposes a limit on the power loads in ITER of $10 \mathrm{MW} / \mathrm{m}^{2}$ at steady state. ${ }^{1}$ During certain limited periods, such as the transients induced by ELMs, higher fluxes can be structurally tolerated but at the cost of increasing the erosion of the tiles. For carbon fiber composite (CFC) tiles, material erosion and the subsequent redeposition could impose strong limitations on the operation of ITER due to tritium being retained in the eroded material. Previous evidence from JET DT campaign in $1997^{2}$ showed that in the most pessimistic case ITER tritium content could reach the limit of $1 \mathrm{~kg}$ in less than 100 discharges. Therefore one of the higher priority activities is to understand the erosion and migration of materials and to characterize them with the present graphite divertor, before moving to a metallic wall. A complete set of diagnostics was therefore developed and installed during the last shutdown to address this issue. ${ }^{3}$ These include sticking monitors, rotating detectors, modified tiles, and mirror test units. A notable innovation is the ability to perform measurements on time scales much shorter than the traditional method with wall probes, which integrate over many discharges and are analyzed only when removed from the machine following the conclusion of an experimental campaign. The most developed diagnostic for this type of time resolved analysis is the quartz microbalance (QMB). ${ }^{4}$ This consists of piezoelectric crystals, which operate as electromechanical transducers, which are located inside a resonating electronic circuit where they vibrate at different frequencies depending on the thickness (i.e., weight) of the deposited layer (see Fig. 1). Hence changes in frequency can be converted directly into mass changes of a layer. Careful calibration in controlled conditions showed that with this approach a resolution of about $15 \%$ of a monolayer can be achieved in JET (where the distance between carbon layers is about $0.24 \mathrm{~nm}$ ). Since the typical oscillation frequency of these crystals is of the order of $6 \mathrm{MHz}$, the transmission of these frequencies inside the vessel through wires poses significant problems. The adopted solution consists of mixing this signal with a reference crystal and the beating frequency, of the order of $1 \mathrm{kHz}$, is more easily transmitted via the vacuum feedthroughs. Another issue is temperature compensation because the oscillation frequency of the crystal is also sensitive to this and so an independent reference crystal is used to compensate for this effect. As it is necessary for the electronic circuit, which performs the first stage signal processing, to be located in- 


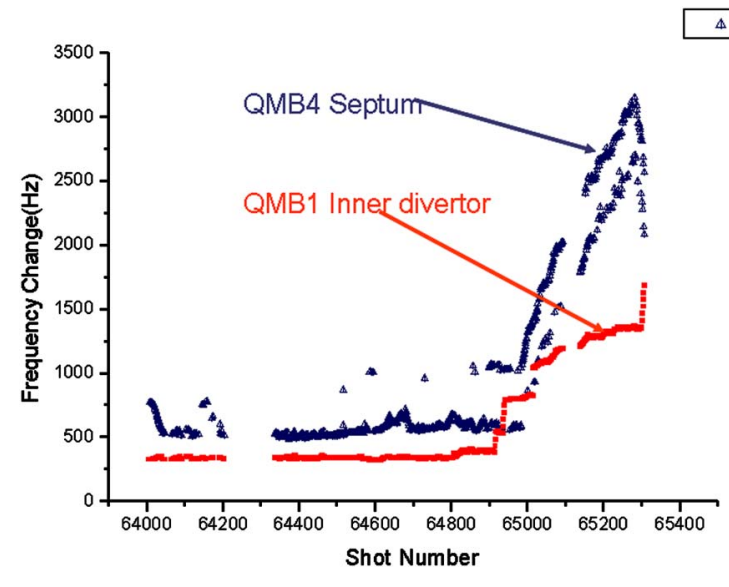

FIG. 2. Quartz microbalance: first signals obtained after opening of the shutters for two new detectors located in the JET divertor.

side the JET vacuum vessel, it therefore has to operate normally at a steady state temperature of up to $200{ }^{\circ} \mathrm{C}$. This requires the use of specially customized integrated circuits, originally developed by the oil exploration industry for operation in harsh environmental conditions.

A prototype QMB worked successfully for about two years in the JET divertor. It was replaced during the last shutdown by a similar "standard" one at the same location. Since the transport and deposition of carbon in JET appear to depend on the substrate temperature, it was considered a strong possibility that the rate of deposition as measured on the crystal of the QMB could depend on its temperature. Accordingly, two other QMBs (design temperature of $300^{\circ} \mathrm{C}$ ) with heated crystals were mounted one in the inner and one in the outer divertor. Of course, the heaters can be switched off, to operate the QMBs in a way equivalent to the "standard" one. Furthermore a cooled QMB was implemented (cooled by connecting part of its body to the inner water-cooled divertor louver structure). All the mentioned QMBs are fitted with shutters, so that measurements can be made for a selected part of a pulse. The signals from two different QMBs are reported in Fig. 2 versus shot number, where the frequency change after opening of the shutter is very evident. These new diagnostics are anticipated to provide much detailed time resolved information during the next campaigns. A new suite of QMB detectors will also be installed during the 2008 shutdown to enable the same studies to be performed with the new full metallic wall.

\section{DIAGNOSTICS FOR MHD INSTABILITIES: MICROWAVE REFRACTOMETRY}

To achieve the goal of a working fusion reactor, it will be necessary to further improve the understanding of MHD instabilities and their impact on the performance of the various plasma configurations. MHD instabilities can indeed strongly influence the behavior of the discharge, degrade the confinement of the fast particles, and even cause mode locking and disruptions. Advanced scenarios, which strongly rely on sophisticated tailoring of the current profile and are therefore close to the instability threshold, could be particularly sensitive to these effects. Over the last few years, consider- able effort has been devoted to the detection of Alfvén cascades (ACs), which have been observed in practically all the major tokamaks. The detection of these ACs is particularly important for the diagnostic of advanced plasma scenarios, because it can help following the time evolution of the minimum of the safety factor, a parameter of crucial relevance for the triggering of internal transport barriers. ${ }^{5}$ Indeed, the characteristic frequency $\omega_{\mathrm{AC}}(t)$ of these modes is linked to the minimum of $q$ by the following relation: ${ }^{6}$

$$
\omega_{\mathrm{AC}}(t)=\left|m / q_{\min }(t)-n\right| V_{A}(t) R_{0},
$$

where $V_{A}$ is the Alfvén velocity, $m$ the poloidal mode number, $n$ the toroidal mode number, and $R_{0}$ the major radius. It was shown on $\mathrm{TFTR}^{7}$ that diagnostics for accessing the plasma core, such as $X$-mode reflectometry, can provide more information on the Alfvén cascades than the external magnetic pickup coils generally used for their detection. Reflectometry determines the effects of the MHD modes on the density fluctuations, therefore providing localized information. Unfortunately the advanced scenarios developed at JET generally can lead to flat density profiles and therefore it is not possible to probe the core and the high field side regions of the plasma with $O$-mode reflectometry, since the probing waves cannot access the internal regions of the plasma. On the other hand, the density fluctuations induced by the Alfvén cascades are mainly localized in the high magnetic field side. These can be accessed by utilizing frequencies, higher than the maximum of the plasma frequency, that are not reflected by the plasma. Waves with these frequencies can cross the whole plasma and can be reflected by the inner wall and then detected by mixing with the signal of the local oscillator, thus performing a refractometric type of measurement. This new approach was developed on JET and proved particularly effective in studying the Alfvén cascades. ${ }^{7}$ It consists of injecting waves at fixed frequency just above the maximum plasma frequency, so that the waves can propagate through the whole plasma but at the same time be very sensitive to the density fluctuations (which is not the case for waves in the typical interferometric regime, i.e., with frequencies much higher than the plasma frequency). To perform these measurements, a ten-channel fixed frequency reflectometer (from 18.6 up to $69.6 \mathrm{GHz}$ corresponding to the critical density range of $\left.(0.43-6) \times 10^{19} \mathrm{~m}^{-3}\right)$ was used, probing the midplane plasma with the $O$-mode polarization. In Fig. 3 a spectrogram [sliding fast Fourier transform (FFT)] of the reflectometer "homodyne" signal $a(t) \times \sin [\phi(t)]$ at frequency $f=45.2 \mathrm{GHz}$ is shown. The Alfvén cascades are detected with high time and frequency resolutions, far better than from other diagnostics such as the magnetic pickup coils. This improvement is seen in Fig. 4, where the Alfvén cascade measurements obtained with the microwave refractometer and other diagnostics are compared.

\section{DIAGNOSTICS FOR ALPHA PARTICLES}

The trace tritium campaign and the forthcoming new ITER-like, ion cyclotron resonant heating, antenna project have motivated the pursuit of a consistent series of diagnostic developments directed around burning plasmas. Particular 


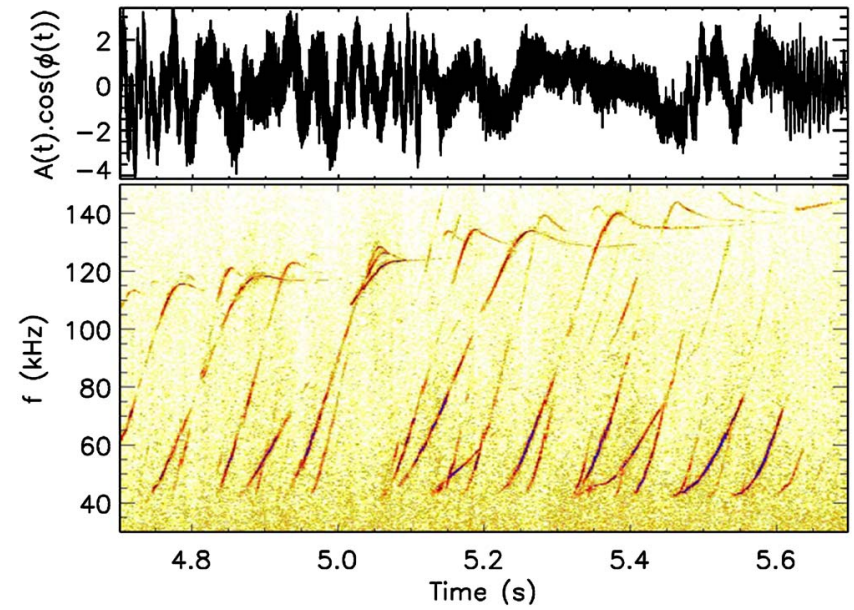

FIG. 3. Observation of different Alfvén cascades from the spectrogram of the homodyne signal (ACs in the $300-400 \mathrm{kHz}$ range).

attention was devoted to diagnosing the alpha particles because, at this stage of the international program and the official launch of the ITER project, it was considered extremely important to start assessing the potential of the various scenarios to confine these particles. Therefore, in addition to a series of developments in the field of neutron diagnostics, as reported in Ref. 8, major progress was achieved in the detection of fast particles. For the highest energy range, gamma-ray detection is now an established tool, reviewed in Ref. 9. However, the lower energy ranges are more problematic. The main wave-particle interactions are expected to take place in the energy range between 50 and $600 \mathrm{keV}$ in JET plasmas. In this interval, the fast ions cannot induce nuclear reactions, which cause the emission of $\gamma$ radiation, because their energy thresholds are above $1 \mathrm{MeV}$. To improve JET diagnostic capability in this respect, a new method is being developed, based on the injection of

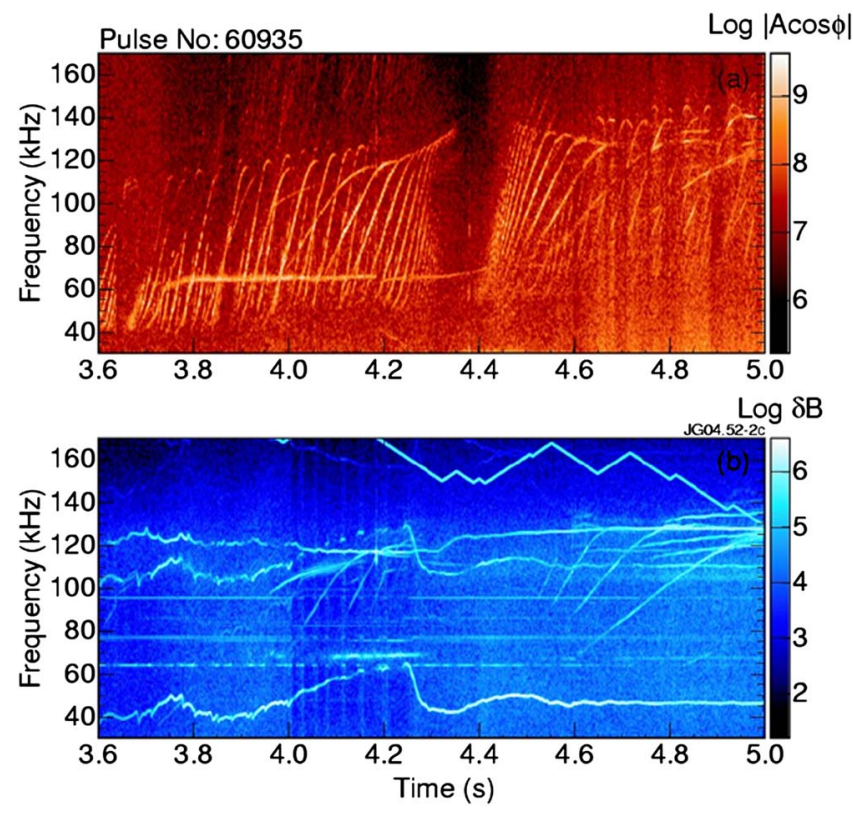

FIG. 4. Alfven cascades detected with the pickup coils (top) and the microwave refractometer (bottom). Toroidal modes numbers up to 16 have been resolved.

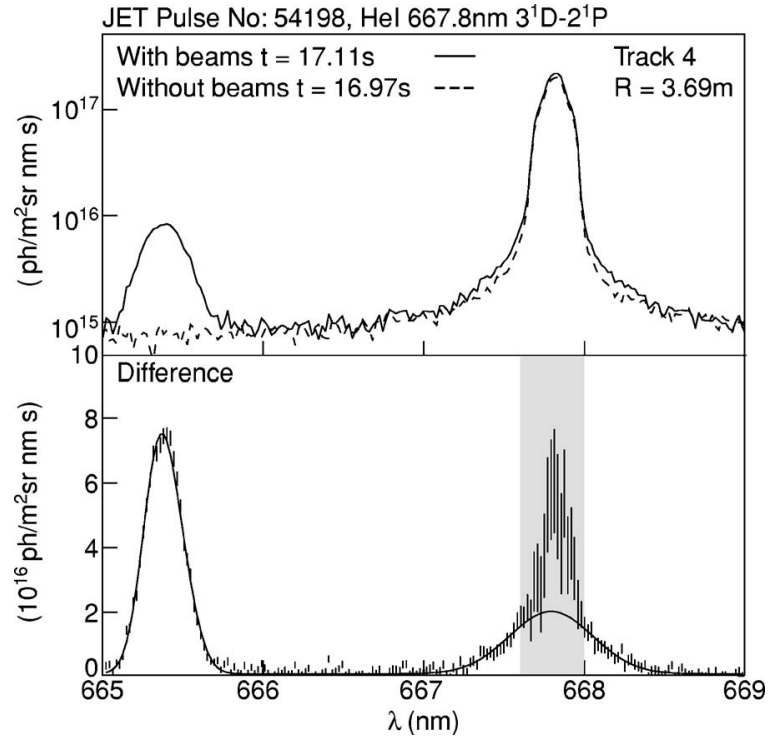

FIG. 5. Signal of double charge exchange. On the top graph are reported the signals with the beam on (continuous line) and with the beam off (dashed line). The bottom graph shows the difference between the two previous curves and the shaded area identifies the part of the signal proportional to the density of neutralized $\mathrm{He}$ (He ash).

extrinsic impurities, such as crypton, whose fluorinelike ionization stage presents transition preferentially populated by fast ions and which emit in the extreme vacuum ultraviolet part of the spectrum. ${ }^{8}$ An even more difficult challenge is posed by the alpha particles once they are thermalized. When they reach a temperature comparable with the plasma bulk, their energy is not sufficient to induce either nuclear reactions or deep atomic transitions and so a more direct method is required. One possible solution is to exploit charge exchange reactions between the thermalized $\mathrm{He}^{2+}$ and beam injected neutral He atoms. In particular, the thermalized alpha particles can undergo a double charge exchange with the beam neutrals which then emit visible radiation after subsequent collisions with the electrons. From the intensity of this emission the density of the emitting ions (He ash) can be estimated. Some preliminaries tests were performed at JET to qualify this approach. ${ }^{10}$ Helium was injected with ${ }^{4} \mathrm{He}$ neutral beams at $29.5 \mathrm{keV} / \mathrm{amu}$ into a nearly $90 \% \mathrm{He}$ plasma. Neutral helium forms a halo of neutral $\mathrm{He}^{0}$ in the ground state and, for $v_{\text {beam }} \gg v_{\text {thermal }}$, the "core plasma" $\mathrm{He}^{0}$ population results from an equilibrium between double charge transfer and ionization processes according to the relation

$$
n_{\mathrm{He}^{2+}} \times n_{\text {beam }} \times \sigma_{2 \mathrm{CX}} \times v_{\text {beam }}=n_{\mathrm{He}^{0}} \times n_{e} \times S,
$$

where $v_{\text {beam }}$ is the velocity of the helium ions in the beam, $n_{e}$ the electron density, $n_{\mathrm{He}^{0}}$ the density of the neutral helium, $n_{\mathrm{He}^{2+}}$ the density of thermalized $\mathrm{He}$ in the plasma, $\sigma_{2 \mathrm{CX}}$ the cross section of the double charge exchange process, and $S$ the ionization cross section. This equation can be expressed in terms of measurable quantities as 


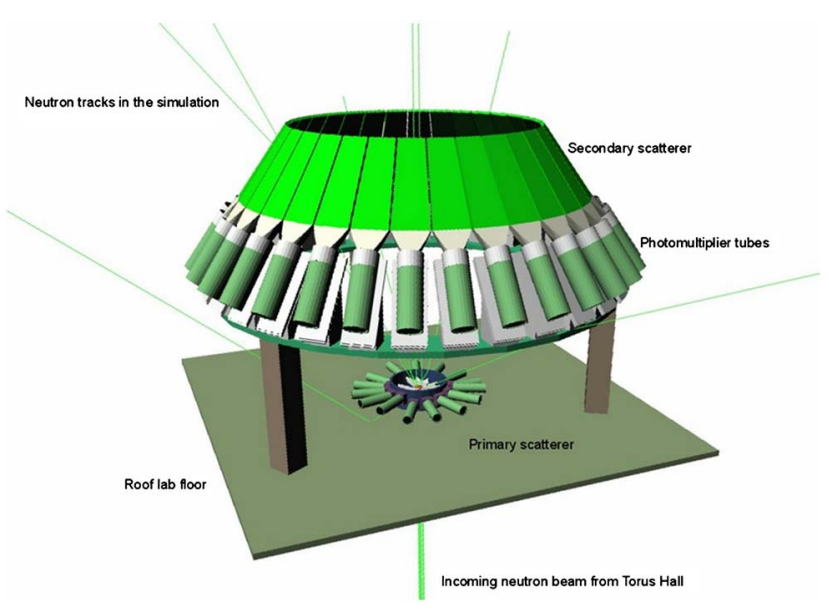

FIG. 6. TOFOR: JET new neutron spectrometer based on the time of flight approach for high counting rate of the $2.45 \mathrm{MeV}$ neutrons.

$$
\frac{I_{\text {thermal }}}{I_{\text {beam }}} \propto \frac{n_{\mathrm{He}_{2+}}}{n_{e}} \frac{X_{\lambda}}{S} \frac{\sigma_{2 \mathrm{CX}} v_{\text {beam }}}{f_{m 1}\langle\sigma v\rangle_{\lambda, m 1}+f_{m 2}\langle\sigma v\rangle_{\lambda, m 2}},
$$

where $f_{m 1}$ and $f_{m 2}$ are the fractions of the beam atoms in the ground state $1 s^{2}{ }^{1} S$ and metastable state $1 s 2{ }^{1} S$, respectively. $X_{\lambda}$ is the collisional radiative coefficient for emission at the wavelength $\lambda_{m 1}$. The radiation was detected using the visible spectrometers of the CXRS system and observing the localized He I $667.8 \mathrm{~nm}$ line about the beam axis. Although this measurement is quite delicate, as can be seen by the small signal shown in Fig. 5, the approach has some potential advantages. Firstly, there is no fast ion contamination because these particles escape from the viewing cones before being excited which confines the technique to measuring just the thermal alphas. Secondly, the emission spectrum is much simpler than the standard single charge exchange. Finally, errors in instruments, absolute calibration, and alignment do not propagate to the $\mathrm{He}^{2+}$ density. Moreover the triplet component of the beam is attenuated in the first centimeters of the edge plasma and therefore uncertainties in the beam density do not affect the accuracy of the measurements in the core.

\section{NEUTRON SPECTROMETRY}

The neutron emission is certainly one of the indispensable parameters to assess the performance of any deuterium or deuterium-tritium hot plasma. The neutrons will provide the net source of energy to heat the primary fluid in the cooling circuit of a future reactor. In an experimental device, such as ITER or JET, in addition to the total yield, it is extremely important to determine also the spectrum of the neutrons, in order to discriminate the percentage due to thermal reactions from other components. To this end, on JET in the last years a lot of efforts have been put into neutron spectrometry. All the major techniques applicable to a tokamak plasma are represented ${ }^{8}$ and significant attention is being devoted to extrapolating the various approaches to ITER. Moreover, given the increasing emphasis of JET program towards the development of ITER scenarios, it was decided to provide the spectrum of the $2.45 \mathrm{MeV}$ neutrons on a regu-

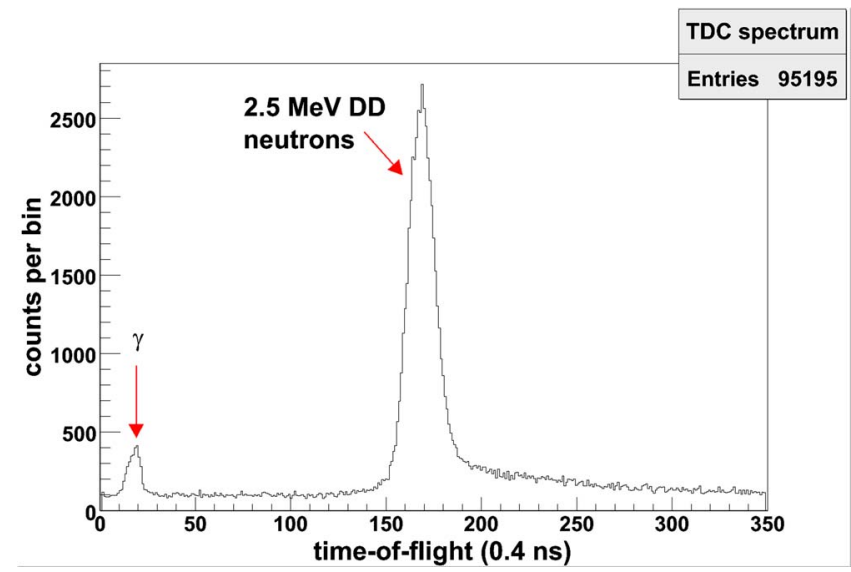

FIG. 7. TOFOR spectrum showing the peak due to the gamma rays and the bigger one generated by the $2.45 \mathrm{MeV}$ neutrons

lar basis. To this end a new spectrometer based on the time of flight approach was installed during the last shutdown. It is called TOFOR since it was explicitly designed to detect the neutron spectra at high counting rate (see Fig. 6). The design of the spectrometer was performed with the help of CERN package GEANT. The calculations required 2.5 years of CPU time, producing a database of 200 Gbytes from which the 2 Gbyte instrumental function was derived. A typical spectrum is reported in Fig. 7 and the diagnostic is expected to reach a peak rate of $30 \mathrm{spectra} / \mathrm{s}$ at 10000 events per spectrum.

\section{DISCUSSIONS}

At JET diagnostic upgrades are fully integrated in the program of scenario development towards ITER. In this respect, particular attention was devoted to some unique aspects of the machine, among which the most relevant are the wall mix of material and the "burning plasma" issues. These were the fields that promoted more innovation in the diagnostic concepts and technologies. In addition to the diagnostics presented in this article, many other developments are under way. Some of the additional fields with more perspectives to promote further innovation of ITER relevance are certainly real time, communication technology, and radiation hard detectors. During the next framework program, JET is expected to install a new Be wall with a W divertor, in addition to upgrading the neutral beam to about $35 \mathrm{MW}$ of power. The scientific issues to be addressed in the light of these improvements will require, among other diagnostics, significant improvements in spectroscopy and IR thermography.

\footnotetext{
${ }^{1}$ ITER Physics Basis editors, Nucl. Fusion 39, 2137 (1999).

${ }^{2}$ P. Andrew et al., J. Nucl. Mater. 266-269, 153 (1999).

${ }^{3}$ P. Coad et al., Fusion Eng. Des. 74, 745 (2005).

${ }^{4}$ H. G. Esser et al., J. Nucl. Mater. 337-339, 84 (2005).

${ }^{5}$ R. Nazikian et al., Phys. Rev. Lett. 91, 125003 (2003).

${ }^{6}$ S. E. Sharapov et al., Phys. Rev. Lett. 93, 165001 (2004).

${ }^{7}$ S. E. Sharapov et al., Phys. Lett. A 289, 127 (2001).

${ }^{8}$ A. Murari et al., Plasma Phys. Controlled Fusion 47, B249 (2005).

${ }^{9}$ V. Kiptily, Rev. Sci. Instrum., these proceedings.

${ }^{10}$ M. O'Mullane et al., Plasma Phys. Controlled Fusion 45, 1747 (2003).
} 